\title{
Dorsal Root Ganglion (DRG) and Chronic Pain
}

\author{
Amnon A. Berger (iD ${ }^{1,}{ }^{*}$, Yao Liu ${ }^{1}$, HarLee Possoit ${ }^{2}$, Anna C. Rogers ${ }^{2}$, Warner Moore $^{2}$, Kyle Gress ${ }^{3}$, Elyse

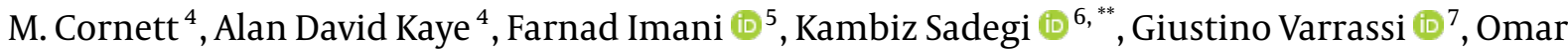 \\ Viswanath (ii) ${ }^{3,8,9,10}$ and Ivan Urits (iD) 3,11
}

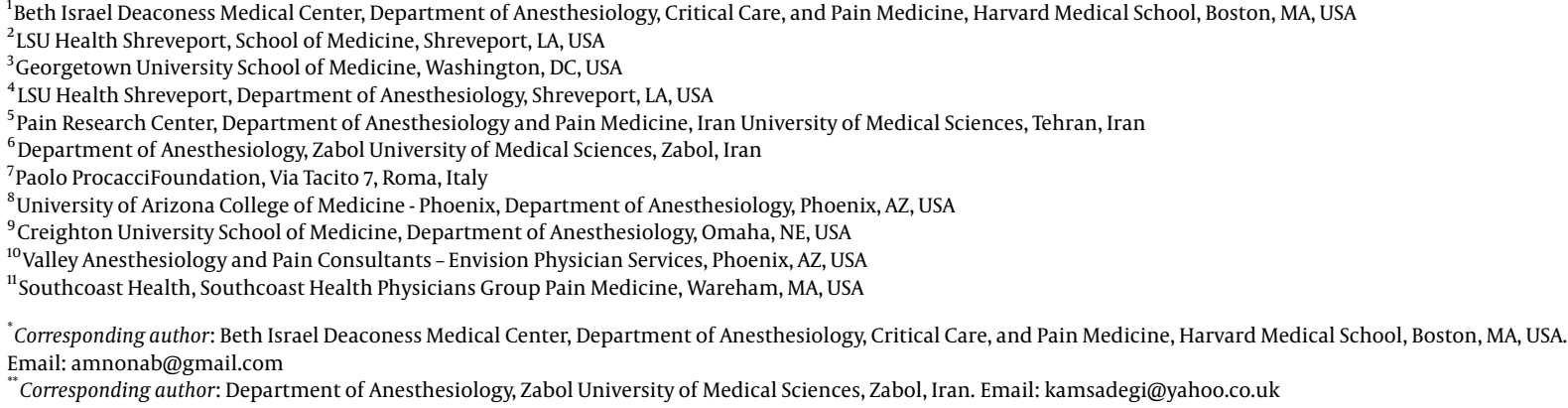

Received 2021 January 18; Revised 2021 March 01; Accepted 2021 March 04.

\section{Abstract}

Context: Chronic neuropathic pain is a common condition, and up to $11.9 \%$ of the population have been reported to suffer from uncontrolled neuropathic pain. Chronic pain leads to significant morbidity, lowered quality of life, and loss of workdays, and thus carries a significant price tag in healthcare costs and lost productivity. dorsal root ganglia (DRG) stimulation has been recently increasingly reported and shows promising results in the alleviation of chronic pain. This paper reviews the background of DRG stimulation, anatomical, and clinical consideration and reviews the clinical evidence to support its use.

Evidence Acquisition: The DRG span the length of the spinal cord and house the neurons responsible for sensation from the periphery. They may become irritated by direct compression or local inflammation. Glial cells in the DRG respond to nerve injury, producing inflammatory markers and contribute to the development of chronic pain, even after the resolution of the original insult. While the underlying mechanism is still being explored, recent studies explored the efficacy of DRG stimulation and neuromodulation for chronic pain treatment.

Results: Several reported cases and a small number of randomized trials were published in recent years, describing different methods of DRG stimulation and neuromodulation with promising results. Though evidence quality is mostly low, these results provide evidence to support the utilization of this technique.

Conclusions: Chronic neuropathic pain is a common condition and carries significant morbidity and impact on the quality of life. Recent evidence supports the use of DRG neuromodulation as an effective technique to control chronic pain. Though studies are still emerging, the evidence appears to support this technique. Further studies, including large randomized trials evaluating DRG modulation versus other interventional and non-interventional techniques, are needed to further elucidate the efficacy of this method. These studies are also likely to inform the patient selection and the course of treatment.

Keywords: Neuropathy, ACNES, Post-herpetic Neuralgia, Ascending Tracts, Interventional Pain Management, Chronic Pain, Dorsal Root Ganglia

\section{Context}

Chronic neuropathic pain is among one of the most widespread complaints of patients, as well as one of the most difficult to treat. Many methods and therapeutic targets have been attempted, with most seeming to have limited benefit and similar outcomes. It has been docu- mented that as many as $11.9 \%$ of general practice patients suffer from inadequately controlled neuropathic pain (1). Further evidence demonstrates an increased suicide rate among these patients, defining the need for improved pain control methods (1). Many approaches have been attempted to target chronic pain, with varying efficacy and risks (2). Pharmacotherapy, which is usually persued fol- 
lowing conservative treatment, is limited in most patients; and importantly, usage of opioid medications can lead to addiction and use disorder (3). Some emerging methods, including continuous infusion of medications such as magnesium and lidocaine are promising, but require further evidence to support their use $(4,5)$. Non-DRG stimulation, such as peripheral nerve stimulation and spinal cord stimulation, has also been shown to be very effective in other conditions, but is less suitable when the DRG is directly involved (6-8). In contrast to existing suboptimal treatment methods, targeting the dorsal root ganglion (DRG) has begun to show promising effects in pain alleviation.

The DRG are highly complex structures located on either side of the spinal cord than span the length of the spinal column. Each ganglion is an enlargement of the dorsal roots given off by the spinal cord. These structures are merely the size of a peanut but are able to house up to 15,000 neurons each. The neurons located within the DRG are responsible for sensory transduction and modulation from the periphery, including pain perception (9). The location of the DRG, which is surrounded by rigid bony structures, leaves little room for expansion of displacement. Herniated disc and osteophytes are common conditions that may cause compression and inflammation at the level of the DRG (10).

The DRG contains clusters of the cell bodies of primary sensory neurons. Each of the axons of these sensory neurons house a variety of fibers with a range of size and excitability. These fibers include the $\mathrm{A} \beta, \mathrm{A} \delta$, and $\mathrm{C}$ fibers. Compared to the large, myelinated, and high velocity $\mathrm{A}$ fibers, the $\mathrm{C}$ fibers are unmyelinated, smaller in diameter and have a much slower conduction velocity. Despite these variations, each of these fibers are responsible for conducting sensory signals from the periphery to the DRG and finally the central nervous system(9). Further research demonstrates $C$ fibers, specifically, play an active role in chronic pain. C-fibers nociceptors have been noted to be involved in aberrant pain signaling within the cell bodies of the DRG (11).

The DRG became a target for pain control, when it was initially hypothesized to be a source of chronic neuropathic pain. Initial therapies targeted towards the DRG were documented as early as 1949. Despite theories of the direct pain control at this location, the difficulty of access or minimal results have limited the progression of DRG pain control. Throughout the years, there have been various methods involving the DRG tested for pain relief. Some of these methods included dorsal rhizotomy or ganglionectomy, dorsal root entry zone (DREZ) lesioning (an adjacent related neural target), conventional radiofrequency denervation, pulsed radiofrequency, and steroid injection $(9,12)$. It has only been within recent years that neuromodulation of DRG, a less invasive measure, has gained greater recognition. Though this type of therapy may be expensive, a cost analysis study showed DRG to be the superior form of treatment when considering cost effectiveness (13).

\section{Evidence Acquisition}

\subsection{Pathophysiology and Mechanisms}

Chronic neuropathic pain often begins with an injury to a peripheral nerve. Once the initial inciting injury takes place, a cascade of inflammatory mediators is activated both peripherally and centrally. Immune cells, including T-cells and macrophages, begin to populate within the dorsal root ganglion that corresponds to the peripherally injured nerve. Studies have shown that these T-cells and major histocompatibility complex class II + macrophages can be found within the DRG even months after the initial injury. One theory states that these persistent inflammatory cells with the ganglion can lead to chronic stimulation of the neurons, leading to the prolonged sensation of pain (9).

The DRG houses neurons that are circumferentially surrounded by supportive glial cells. Normally, these glial cells provide protection for the neurons. However, following injury, the glial cells can also release immune response cells, further attenuating the inflammatory response at the DRG. It is also reported that injury to the peripheral nerves can cause the release of neurotropic factors within the glial cells. These factors cause a growth response of the glia, triggering mechanical compression of the sensory neuron cell bodies. This compression causes further irritation of the nerves within the DRG, which contributes to the persistent cascade of immune cells and inflammatory reaction. These factors collectively compile to create a cycle of inflammation within the DRG, even after the peripheral nerve injury has completely healed (9).

Peripheral sensory neurons rely primarily on voltagegated calcium channels to propagate action potentials, including those within the DRG. Regulation of these channels has been hypothesized to play a role in chronic pain, as well. During the conduction of an action potential, Nand T-type calcium channels activate and begin to increase their permeability to allow the influx of more calcium. This increase in intracellular calcium concentrations signals a 
cell-specific cascade of signals. In regard to chronic pain, these cells may have become hyperexcitable to the presence of calcium. This hyperexcitability leads to further sensitization of the cells and constant stimulation of the neurons within the DRG, furthermore leading to the chronic pain perception (1).

Among the many treatment options attempted, DRG stimulation is one that has provided the most success. The theory behind the viability of this treatment involves voltage-gated calcium channels, as well. It is thought that exogenous stimulation at the level of the DRG interrupts the aberrant pain signaling taking place. When the external impulse is introduced, it is hypothesized to cause a "stimulation-induced action potential failure (14)." The blocking of the action potential signaling will then effectively prevent the pain signal from reaching the central nervous system.

On the molecular level, epigenetic modifications have recently come to light concerning their role in chronic pain (15). In a study, where DRG neuropathic pain was induced there was correlation with specific histone acetylation. It was demonstrated that acetylation at the $\mathrm{H} 3$ and $\mathrm{H} 4$ histones is involved in the maintenance of chronic pain. Although these conclusions are still controversial, it is yet another option for targeting pain directly at the DRG (15). Another target that is more commonly used is that of the KCNQ potassium channels. These channels are found within the nociceptive DRG neurons and have been hypothesized to decrease the excitability of the neurons. When these channels are activated, the potassium currents effectively dampen the action potential of an approaching pain signal. Common neuropathic pain drugs including gabapentin and retigabine have been known to work via this mechanism (11).

There are several theories behind the chronic neuropathic pain and the source of said pain. As more studies have been completed, the dorsal root ganglion has proven to be the more promising source to approach for directed pain control. As the DRG sits physically and physiologically at the junction of the peripheral and central nervous system, these structures provide an ideal target for pain control.

\subsection{Anatomy and Techniques to Stimulate the DRG}

The spinal cord is made up of 31 pairs of spinal nerves composed of afferent sensory dorsal and motor ventral roots. The dorsal root exits from the intervertebral foramina and merges with the ventral nerve root to form the dorsal root ganglia (DRG). The DRG is a bundle of pseu- dounipolar neurons located in the dorsal root of the spinal nerve roots which serve in the development and maintenance of neuropathic pain and change in sensation (10). The unique structure of the pseudounipolar neuron contains two branches. The first branch forms a T-junction to the periphery, and the second extends toward the spinal cord acting as a low-pass filter. These neurons participate in the propagation or impeding of an electrical pulse (10). Due to a multitude of anatomic variations, the size and location of the DRG can vary with increasing length and width inside the lumbar regions L4 -L5 as well as between the neuroforaminal, intraspinal, and extraforaminal regions (10). This variation can lead to DRG compression and entrapment, leading to an abundance of pathologies such as disc herniations and osteophyte formation, ultimately causing chronic pain within the affected dermatomes. The lumbar region DRGs play a key role in the occurrence of low back pain and sciatica and are highly sensitive to mechanical compression (16). The DRG is also thought to play a significant role in other chronic pain conditions such as postherpetic neuralgia, chronic visceral pain, and peripheral neuropathies. Targeted neuromodulation of the DRG has been shown to be an important therapeutic option for chronic pain management due to its participation in the development and maintenance of neuropathic pain (17). Many different techniques have reported significant relief from DRG stimulation. DRG stimulation must be carefully mapped and evaluated to ensure that the correct location and region will be specifically targeted to gain a therapeutic effect (17-19).

\subsection{1. "Transgrade" Epidural Technique}

Costanzi et al. describe the use of transgrade epidural DRG lead placement for modulation. The transgrade approach allows for placement of a lead in the neuroformaina opposite of the interlaminar space which has may have operational and long-term benefits (17). Fluoroscopy is used to identify the appropriate level. The entry point was marked over the contralateral superior articular process of the corresponding vertebrae (17). A $2 \mathrm{~cm}$ incision is made over the entry point, dissecting down to the prevertebral fascia making a small pocket for anchoring and allowing for insertion of a 14G RX 2 Coudé epidural needle aiming for the midline epidural space. After loss of resistance the needle is angled posteriorly for lead placement inside the dorsal foramina. A Linear ST Percutaneous Lead 8 electrode subcompact lead of $31 \mathrm{~mm}$ with contact electrodes of 3mm, edge-to-edge width of $1 \mathrm{~mm}$ and diameter of $1.3 \mathrm{~mm}$ is then used with conformation of placement via 
lateral imaging (17). Anchoring of the leads is secured to the prevertebral fascia allowing extensions to be attached and tunneled out for attachment to an external pulse generator. In their publication, Costanzi et al. used stimulation parameters of a pulse rate of $70 \mathrm{~Hz}$, pulse width of 150 $\mu$ s and incremental amplitude in steps of $0.1 \mathrm{~mA}(17) .39$ patients underwent a trial of DRG stimulation with an implantation rate of $82 \%$. The results of the study were based on Numeric Rating Scale (NRS-11) score and Patient Global Impression of Change (PGIC). Successful treatment with DRG stimulation was defined as a decrease of NRD-11 score $>2$ from baseline before the trial and an average weekly score taken after 6 weeks and a PGIC score of 6 or 7 which was based from a stemmed question about their pain, activity, emotion, and overall quality of life (17). The rate of successful treatment, according to NRS-11 and PGIC scores, was $87 \%$ at 6 weeks and $66 \%$ at a mean of 18 months followup (17). The most common complication was pain at the site of the pocket made for anchoring and lead migration.

\subsubsection{Selective Radiofrequency Technique}

Davis et al. utilize radiofrequency (RF) energy for the selection of the DRG's, which correlated to the areas patients were reporting pain. A modified, transforaminal epidural approach targeting the posterior aspect of the foramen, directly inferior to the pedicle, was used for the placement of 20 -gauge $100 \mathrm{~mm}$ conventional RF cannulas with $5 \mathrm{~mm}$ curved active tips (20). The modification of obliquing the C-arm $10^{\circ}-15^{\circ}$ farther allowed for the cannula entry point to be "flatter" when reaching the foramen. The tips of the cannulas were advanced to the medial aspect of each pedicle. The RF generator stimulated the nearby DRG via the "sensory testing" function. The energy was increased until the patient reported feeling the sensation within the particular area, whether it covered the painful area, and how much of the area. Three to four DRGs were stimulated through the same method until the patient reported stimulation to all areas with pain. Once the DRG was mapped, Slim Tip Axium leads were placed using the technique previously described by Liem et al., which utilizes quadripolar neurostimulation leads. Leads were placed via an epidural approach advancing the leads in an anterograde fashion and steering into the intervertebral foramen near the DRG under fluoroscopic guidance (18). The four patients included in this one-week trial reported 60 - 90\% pain relief upon completion (20). Coverage of pain relief included painful areas and were scheduled for permanent implantation.

\subsubsection{Percutaneous DRG Wireless Technique}

The stimwave technologies incorporated freedom SCS system was utilized by Speck et al. This system is comprised of an implantable stimulator with four electrodes and a transmitter worn externally. The stimulator acts on the nerves to inhibit signal transmission from the DRG to the brain by utilizing pulsed electrical currents. The stimulator is transforaminally placed at the DRG. The study was completed in two phases. In the first phase, the stimulators were not anchored, whereas in the second phase, a wing anchor was used to fix the stimulator unilaterally in the axilla of the targeted foramen, which contained the traversing and exiting nerves (19). The anchor was provided as part of the dispensing tool, and once the stimulator was in the desired location, the anchor and dispensing tool are placed over the stimulator for the anchor to be "deployed" percutaneously. The inner canula of the dispensing tool was threaded over the stimulator for the dispensing tool's tip to puncture the tissue around the stimulator to the desired depth (19). The anchor is placed around the stimulator and cinched tightly to decrease the likelihood of migration. The built-in receiver of the system communicates through the skin and is controlled via the externally worn transmitter to provide the power and stimulation parameters using a proprietary pulse modulation and pulse width modulation scheme (19). The results showed an average of overall pain reduction was $59.9 \%$ regardless of the device placement in Phase 1 and 2, covering only a portion of the painful areas. The average threshold of stimulation perception was reported as $1.26 \mathrm{~V}(0.5-2.0 \mathrm{~V})$ (19). Good to excellent overall pain relief was reported by 7 of 11 patients (63\%), 2 reported fair overall intensity pain relief, and 2 reported poor or no overall pain relief, which was primarily due to migration during Phase 1 (19). Phase 1 subject stimulators migrated an average distance of $8.80 \mathrm{~mm}$, indicating a need for an anchor in Phase 2, which decreased the migration of the stimulator to an average of $1.83 \mathrm{~mm}$.

\subsection{Risk Factors and Safety}

Stimulation of the DRG has several advantages for achieving neuromodulation for the treatment of a wide range of indications such as refractory pain, postsurgical pain, complex regional pain syndrome, and phantom pain. Accessing DRGs allow for targeting of selective and specific sets of sensory afferent fibers within their vertebral segment for chronic pain modulation. The limited cerebrospinal fluid around the DRG allows closer proximity of the electrical stimulation allowing for more uniform stimulation and amplitude to the affected area (21). 
This close proximity of the electrode to the DRG also allows for a reduction of electrode movement with postural change, decreasing the chance of over or under stimulation (21). Some factors that account for the differences in outcomes described with DRG stimulation are anatomical differences in the DRG leading to a difference in body surface coverage and postural changes. Analysis by Deer el al. suggests that DRG stimulation allows more analgesia without the underlying paresthesia. Factors that resulted in paresthesia are stimulation amplitudes and frequencies, number of implanted leads, and younger age (22). Although analgesia without paresthesia was perceived 60 $70 \%$ of the time in subjects, there are limitations to this observation since investigators gained experience and recognized that subthreshold stimulation could be used, which may have led to more subjects programmed at this setting (22). Also, sensory adaptations to the stimulation could have been achieved. Complications that can arise from the use of DRG stimulation occur in 3 categories, the operator, the hardware, or the patients. The operator error stems from the origin of the DRG stimulation technique, which is relatively new. This technique is more technically demanding and is associated with a learning curve that required a specific skill set (23). Procedural complications include outcomes such as dural punctures, new neurological symptoms with new or worsening radiculopathy being the most common, and hematomas, which could also be related to the device itself (24). The hardware design has been changed for improvement in the past and is likely to be changed again with time due to the occurrence of complications. Some complications that have occurred due to design are lead connection failure, erosion, and difficulty with insertion or removal. Other complications that have been associated with the device are infection, temporary motor stimulation, cerebrospinal fluid leaks, implant site pain, lead migration and lead fracture (23). Infection was the most frequently noted complication with placement of DRG stimulators having an event rate at $1.08 \%$ (25). All other complications of implantation had event rates of $<1 \%$ with cerebrospinal fluid leaks and device related pain with an event rate of $0.54 \%$ (25). Lead migration is also one of the most frequently reported complications due to the difference in anchoring methods. In the traditional spinal column stimulation(SCS) system, it is anchored to the supraspinous ligament or fascia, which is less commonly used in DRG lead implantation. Although traditional anchoring and strain relief loops are advised, many providers rely solely on strain relief loops, which are created within the epidural space by manipula- tion of the lead after placement overlying the DRG (24). A unique indication with implant site pain with DRG stimulation compared to SCS is the close proximity of the lead to the DRG, which allows lower amplitudes to create overstimulation (24). Patient complications or complaints include outcomes such as anaphylactic reactions, cardiovascular changes, seizures, poor patient selection criteria, and placebo effects. Anaphylactic reactions, cardiovascular changes, and seizures have a very low event rate occurring at rates of $0.09 \%, 0.04 \%$, and $0.04 \%$ (25). Patients with failed back surgery syndrome (FBSS) who were treated with DRG stimulation have shown to be less successful due to buildup of epidural scar tissue making lead placement difficult (23). The high failure rate suggests that patients of this nature are not good candidates for this therapeutic strategy and should not be chosen as subjects. Although complications from DRG stimulation do occur, the clinical adverse events and device complaints were comparable or less frequent than those reported with similar epidural spinal cord neurostimulation systems and similar SCS systems from the same manufacturer (25). The majority of the complications were managed surgically rather than explant, which could suggest that the patient had sufficient initial therapeutic effects (24). Many studies that have been published thus far imply that intervention may be helpful, but the results should be viewed with caution due to a multitude of limitations. Examples of limitations include poor quality of available studies, a small number of participants, a highly selected patient population, conflict of interest of sponsors and authors (26). Small numbers of participants can lead to results that lower the reliability of the study. Highly selected patient populations can introduce bias into the study. Ensuring the validity of the study should include observation of authors reporting attrition during the trial and follow-up periods and intention-totreat analysis when reporting results as well as exclusion or failure of treatment. Most studies with financial support were funded by companies in the industry with commercial interest in neuromodulation with electrical field stimulation of DRG (26). The potential conflicts of interest warrant caution when interpreting results and assessing safety.

\subsection{Clinical Evidence}

Table 1 summarizes the clinical evidence regarding the use of DRG stimulation in chronic back pain, which is reviewed here. 


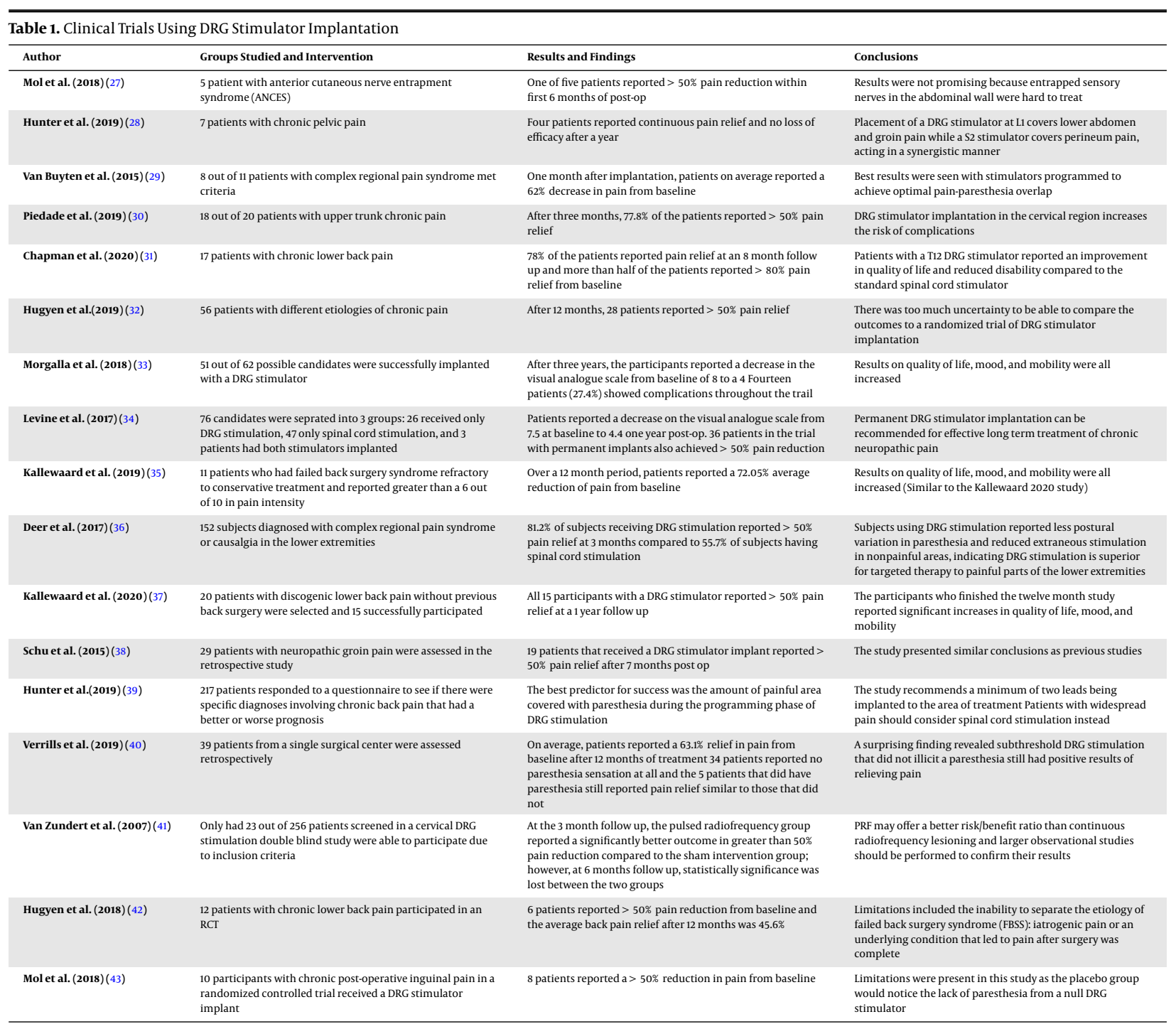

\subsubsection{Case Reports/Studies}

Five patients with anterior cutaneous nerve entrapment syndrome (ACNES) were referred for permanent dorsal root ganglion (DRG) stimulator implantation. The results were not promising because ACNES involves entrapped sensory nerves in the abdominal wall, making it difficult to treat. Only one of the five patients reported greater than 50\% pain reduction within the first 6 months post-op according to a baseline numeric pain score (27).

One novel approach in DRG stimulation assessed by Hunter et al. (2019) was placing simultaneous leads bilaterally in spinal cord levels L1 and S2 to treat chronic pelvic pain. Since the pelvic floor is a complex web of nerve fibers traversing throughout the pelvis, multiple areas were stimulated in order to fully cover chronic pelvic pain from numerous etiologies. Placement of a DRG stimulator at L1 covers lower abdomen and groin pain while an S2 stimulator covers perineum pain, acting in a synergistic manner. Even though it was a small sample size, all seven patients undergoing the study admitted to taking lower dosages of opioid medications, improvement in sexual function, and less frequent urination (28). In another study, eleven patients fitting the criteria for DRG stimulation with complex regional pain syndrome participated in a DRG stimulation trail. Eight of them underwent implantation, and the stimulators were programmed to achieve optimal pain-paresthesia overlap. One month after implantation, patients on average reported a $62 \%$ decrease in pain from baseline while reducing the area of pain distributions (29). 
Postherpetic neuralgia (PHN) has historically been difficult to treat. After conducting a case study, it was found that direct stimulation of electrodes to the affected ganglion resulted in immediate pain, but interestingly placing the electrodes on overlapping dermatomes adjacent to the affected DRG suppressed PHN pain. Out of 20 patients sampled, 18 were approved for permanent stimulation implantation. After three months, 77.8\% of the patients reported greater than $50 \%$ pain relief (30). One key point to be aware of is surgical implantation in the upper trunk requires modification and creates more complications compared to implantation in the lumbar region.

A different study focused on the lumbar spine as the area of interest in treating chronic lower back pain with a T12 DRG stimulator surgically implanted. Out of 17 patients, 78\% of them reported lower back pain relief at an 8 month follow up, and more than half of them reported greater than $80 \%$ pain relief from baseline (31). Most importantly, patients with a T12 DRG stimulator reported an improvement in the quality of life and reduced disability compared to the standard spinal cord stimulator.

\subsubsection{Prospective/Cohort}

A prospective study analyzed 56 patients undergoing permanent DRG stimulator implantation, and after 12 months, 28 patients reported greater than 50\% pain relief (32). Due to the numerous etiologies of chronic pain, there was too much uncertainty to be able to compare the outcomes to a randomized trial of DRG stimulator implantation. Better results were reported in a study involving 62 patients as possible candidates for therapy, and 51 of them were successfully implanted with a DRG stimulator. Results were recorded continuously for a three-year period, a unique variable not many other studies accomplished. After three years, the participants reported a decrease in the visual analog scale from a baseline of eight to a four (33). Fourteen patients (27.4\%) showed complications throughout the trial.

Out of 132 patients trialed in a separate cohort study, 76 patients proceeded to a permanent implant. Patients were separated into three groups based on chronic pain location: 26 patients received only DRG stimulation, 47 patients received only spinal cord stimulation, and 3 patients had both stimulators implanted. Patients reported a decrease in the visual analog scale from 7.5 at baseline to 4.4 one-year post-op (34). 36 patients in the trial with permanent implants also achieved greater than $50 \%$ pain reduction as well. One study explored the effectiveness of DRG stimulation in patients with failed back surgery syn- drome refractory to conservative treatment, who also reported greater than a 6 out of 10 in pain intensity. Eleven patients were implanted with a permanent DRG stimulator, and over a twelve-month period, patients reported a 72.05\% average reduction of pain from baseline (35).

In the Deer et al. study, DRG stimulation had a greater treatment success rate than patients using a spinal cord stimulator. $81.2 \%$ of subjects who received DRG stimulation reported greater than $50 \%$ pain relief at 3 months compared to $55.7 \%$ of subjects with spinal cord stimulation (36). Subjects using DRG stimulation reported reduced extraneous stimulation in nonpainful areas and less postural variation in paresthesia, indicating DRG stimulation is superior for targeted therapy to painful areas of the lower extremities. Device-related and serious adverse events between the DRG stimulation and spinal cord stimulation groups studied were similar. Twenty patients with discogenic lower back pain without previous back surgery were selected for a trial of DRG stimulation, investigating its' significance in providing pain relief. 15 patients with a permanent L2 DRG stimulator implantation reported greater than $50 \%$ pain relief. By using stringent criteria to participate in the trial, selective bias should be considered when interpreting the results. 14 of the participants finished the twelve-month study, and all of them reported significant increases in quality of life, mood, and mobility (37).

An interesting finding in the Morgalla et al. study was the restoration of laser evoked potentials (LEP) by DRG stimulation, which correlated with improved pain relief (44). Scientists hypothesized that peripheral damage to nerves could cause an ectopic firing of the DRG, leading to the activation of diffuse noxious inhibitory control in the dorsal horn and impairment of LEP at the cortical level. With the use of DRG stimulation, high-frequency action potentials were restored to normal as well as pain processing modulation at the cortical level (44).

\subsubsection{Retrospective}

A study assessing neuropathic groin pain reached similar conclusions as previous studies, stating DRG stimulation allows for more precision in the area of chronic pain. Physicians should be informed on the possibility of cerebrospinal fluid causing the unwanted spread of stimulation in dorsal columns at T11-L1, so it is better to place leads at the level of L1-L2 for DRG stimulation (38).

In a separate retrospective study, 217 patients responded to a questionnaire to see if there were specific diagnoses involving chronic back pain that had a better or worse prognosis. The best predictor for success, according 
to this study, was the extent of painful area covered with paresthesia during the programming phase of DRG stimulation (39). Pain in a specific peripheral nerve responded positively, and there was no specific dermatome that responded better than another as long as the pain was localized. The study also recommends a minimum of two leads being implanted into the area of treatment. Patients with widespread pain should consider spinal cord stimulation instead.

A surprising finding in the Verrills et al. study revealed that subthreshold stimulation not eliciting paresthesia still had positive results in pain relief. This suggested several different mechanisms of action for DRG stimulation, including inhibition of supraspinal regions involved in somatic paresthesia sensation. Out of the 39 patients from a single surgical center, the average patient-reported $73.9 \%$ pain relief from baseline after the trial period, and pain relief dropped to $63.1 \%$ after 12 months of treatment. 34 patients reported no paresthesia at all, and the 5 patients that did have paresthesia still reported pain relief similar to those that did not (40). Therefore, more studies should be done to explore paresthesia independent DRG stimulation for chronic pain relief.

\subsubsection{Randomized Controlled Trials}

A cervical DRG double-blind study had only 23 out of 256 patients participate due to inclusion criteria. Patients were randomly assigned to either the pulsed radiofrequency (PRF) group or the sham intervention group. At the 3 month follow-up, the pulsed radiofrequency group reported a significantly better outcome in greater than $50 \%$ pain reduction compared to the sham intervention group; however, at 6 months follow-up, statistical significance was lost between the two groups (41). It established that PRF and sham intervention provide different biological effects, PRF's mode of action is temperature independent, and neural destruction of PRF is dependent on the distance of electrode from tissue. It was concluded that PRF might offer a better risk/benefit ratio than continuous radiofrequency lesioning, and larger observational studies should be performed to confirm these results.

A factor hard to control for in the randomized controlled trial (RCT) done by Hugyen et al. (2018) was the inability to separate the etiology of failed back surgery syndrome (FBSS): iatrogenic pain or an underlying condition that led to pain after surgery was complete. The physicians focused on stimulating the L2-L3 level in patients. 6 out of 12 patients reported greater than $50 \%$ pain reduction from baseline, and the average back pain relief after
12 months was $45.6 \%$ (42). The physicians believed they had good results from the L2-L3 DRG stimulation through synergistically combining segmental and nonsegmental mechanisms of action through the primary sensory afferent and sympathetic pathways (42).

A final randomized controlled trial showed promise with the use of DRG stimulation for chronic postoperative inguinal pain. 8 out of the 10 patients reported a greater than $50 \%$ reduction in pain from baseline (43). One note readers should be aware of are the limitations for a true randomized controlled trial, as a placebo group would notice the lack of paresthesia from a null DRG stimulator. However, on the contrary, DRG stimulator induced paresthesia lets the observer know whether or not the lead is placed in the correct area.

\subsubsection{Meta-analysis}

Pain at the DRG stimulator pocket site was the biggest complication reported with DRG stimulation while treating chronic pain (45). The meta-analysis performed by Hugyen et al. (2020) is consistent with other publications previously reported. There is a similar overlap between all the papers, including inclusion criteria for trials, duration of the study, and potential complications. To assess effectiveness of DRG stimulation, there had to be uniform measures set to compare amongst participants. The scores assessed included numeric pain rating scale (NPRS) and brief pain inventory (BPI), quality of life (EQ-5D score), Oswestry disability index (ODI), and mood scores (Profile of Mood States). Complications in all of these papers are related to lead displacement, pain at the battery site, overstimulation, and lead breakage. A continuous theme in these studies has been the lack of concrete biological evidence of efficacy for DRG stimulation and the struggle of limiting subjective bias in pain relief.

\section{Conclusions}

Chronic pain affects a large part of the population and carries a significant price tag in terms of quality of life, morbidity, and economic loss. Unfortunately, a significant number of patients continue to experience pain with their treatment continues to challenge providers. Many different approaches from multiple disciplines are being researched for the treatment of chronic pain, including conservative, pharmacologic, psychologic, and interventional approaches. Recently, stimulation of the DRG has emerged as a possible technique with positive results.

Housing the neurons that carry sensation from the periphery, direct stimulation of the DRG could mask those 
signals and break off the connection carrying pain signals. The actual mechanism responsible for pain alleviation from DRG stimulation is still being explored, and several molecular patterns, including calcium flux modulation, KCNQ channels, and histone deacetylation, have been suggested.

DRG stimulation is a developing scientific technique with little known about the effectiveness and possible complications of permanent implantation devices to treat chronic pain. However, from the limited studies performed, there have been promising results. Chronic pain can be caused by numerous etiologies, including postherpetic neuralgia, lower axial back pain, chronic pelvic pain, anterior cutaneous nerve entrapment syndrome, complex regional pain syndrome, and many more. Dorsal root ganglion stimulation demonstrated greater improvements in quality of life and psychological disposition. An advantage of DRG stimulation is the precision of stimulation at a particular dermatome in contrast to spinal cord stimulation, which affects a broader region. Another benefit of DRG stimulator implantation is a significant decrease in lead repositioning compared to SCS, allowing for more accurate results when reporting pain.

\section{Footnotes}

Authors' Contribution: Study concept and design: AAB, YL, HP, ACR, WM; Analysis and interpretation of data: KG, EMC, ADK, FI, KS; Drafting of the manuscript: ADK, FI, KS, GV, OV, IU; Critical revision of the manuscript for important intellectual content: AAB, YL, HP, ACR, WM, KG, EMC, ADK, FI, KS, GV, OV, IU; Statistical analysis: AAB, YL, HP, ACR, WM, KG, EMC, ADK, FI, KS, GV, OV, IU.

Conflict of Interests: The authors have no conflicts of interest to disclose.

Funding/Support: No funding was received for the completion of this manuscript.

\section{References}

1. Liem L, Van Dongen E, Huygen FJ, Staats P, Kramer J. The dorsal root ganglion as a therapeutic target for chronic pain. Reg Anesth Pain Med. 2016;41(4):511-9. doi: 10.1097|AAP.0000000000000408. [PubMed: 27224659].

2. Patel VB, Wasserman R, Imani F. Interventional therapies for chronic low back pain: A focused review (efficacy and outcomes). Anesth Pain Med. 2015;5(4). e29716. doi: 10.5812/aapm.29716. [PubMed: 26484298]. [PubMed Central: PMC4604560].

3. Malik KM, Imani F, Beckerly R, Chovatiya R. Risk of opioid use disorder from exposure to opioids in the perioperative period: A systematic review. Anesth Pain Med. 2020;10(1). e101339. doi: 10.5812/aapm.101339. [PubMed: 32337175]. [PubMed Central: PMC7158240].
4. Tully J, Jung JW, Patel A, Tukan A, Kandula S, Doan A, et al. Utilization of intravenous lidocaine infusion for the treatment of refractory chronic pain. Anesth Pain Med. 2020;1(6). e112290. doi: 10.5812/aapm.112290.

5. Urits I, Jung JW, Amgalan A, Fortier L, Anya A, Wesp B, et al. Utilization of Magnesium for the treatment of Chronic Pain. Anesth Pain Med. 2021;11(1). e112348. doi: 10.5812/aapm.112348.

6. Berger AA, Urits I, Hasoon J, Gill J, Aner M, Yazdi CA, et al. Improved pain control with combination spinal cord stimulator therapy utilizing sub-perception and traditional paresthesia based waveforms: A pilot study. Anesth Pain Med. 2021;11(1). e113089. doi: 10.5812/aapm.113089.

7. Urits I, Schwartz R, Smoots D, Koop L, Veeravelli S, Orhurhu V, et al. Peripheral neuromodulation for the management of headache. Anesth Pain Med.2020;10(6). e110515. doi: 10.5812/aapm.110515.

8. Abejon D, Vancamp T, Monzon EM. A cost-consequence analysis examining the differences between non-rechargeable and rechargeable systems. Anesth Pain Med. 2020;10(1). e100308. doi: 10.5812/aapm.100308. [PubMed: 32337173]. [PubMed Central: PMC7158244].

9. Esposito MF, Malayil R, Hanes M, Deer T. Unique characteristics of the dorsal root ganglion as a target for neuromodulation. Pain Med. 2019;20(Suppl 1):S23-30. doi: 10.1093/pm/pnz012. [PubMed: 31152179]. [PubMed Central: PMC6544557].

10. Vancamp T, Levy RM, Pena I, Pajuelo A. Relevant anatomy, morphology, and implantation techniques of the dorsal root ganglia at the lumbar levels. Neuromodulation. 2017;20(7):690-702. doi: 10.1111/ner.12651. [PubMed: 28895256].

11. Wu Z, Li L, Xie F, Du J, Zuo Y, Frost JA, et al. Activation of KCNQ channels suppresses spontaneous activity in dorsal root ganglion neurons and reduces chronic pain after spinal cord injury.J Neurotrauma. 2017;34(6):1260-70. doi: 10.1089/neu.2016.4789. [PubMed: 28073317]. [PubMed Central: PMC5359643].

12. Imani F. Using pulsed radiofrequency for chronic pain. Anesth Pain Med. 2012;1(3):155-6. doi: 10.5812/kowsar.22287523.4047. [PubMed: 24904784]. [PubMed Central: PMC4018683].

13. Mekhail N, Deer TR, Poree L, Staats PS, Burton AW, Connolly AT, et al. Cost-effectiveness of dorsal root ganglion stimulation or spinal cord stimulation for complex regional pain syndrome. Neuromodulation. 2020. doi: 10.1111/ner.13134. [PubMed: 32153073].

14. Kent AR, Min X, Hogan QH, Kramer JM. Mechanisms of dorsal root ganglion stimulation in pain suppression: A computational modeling analysis. Neuromodulation. 2018;21(3):234-46. doi:10.1111/ner.12754. [PubMed: 29377442].

15. Liang L, Tao YX. Expression of acetyl-histone $\mathrm{H} 3$ and acetyl-histone $\mathrm{H} 4$ in dorsal root ganglion and spinal dorsal horn in rat chronic pain models. Life Sci. 2018;211:182-8. doi: 10.1016/j.lfs.2018.09.029. [PubMed: 30236868].

16. Arslan M, Comert A, Acar HI, Ozdemir M, Elhan A, Tekdemir I, et al. Nerve root to lumbar disc relationships at the intervertebral foramen from a surgical viewpoint: An anatomical study. Clin Anat. 2012;25(2):218-23. doi: 10.1002/ca.21213. [PubMed: 21671286].

17. Al-Kaisy A, Royds J, Costanzi M, Racz G, Wesley S, Palmisani S, et al. Effectiveness of "transgrade" epidural technique for dorsal root ganglion stimulation. A retrospective, single-center, case series for chronic focal neuropathic pain. Pain Physician. 2019;22(6):601-11. doi: 10.36076/ppj/2019.22.601.

18. Liem L, Russo M, Huygen FJPM, Van Buyten JP, Smet I, Verrills P, et al. A multicenter, prospective trial to assess the safety and performance of the spinal modulation dorsal root ganglion neurostimulator system in the treatment of chronic pain. Neuromodulation. 2013;16(5):471-82. discussion 482. doi: 10.1111/ner.12072. [PubMed: 23668228]. 
19. Weiner RL, Yeung A, Montes Garcia C, Tyler Perryman L, Speck B. Treatment of FBSS low back pain with a novel percutaneous drg wireless stimulator: Pilot and feasibility study. Pain Med.2016;17(10):1911-6. doi: 10.1093/pm/pnw075. [PubMed: 27125284].

20. Hunter CW, Yang A, Davis T. Selective radiofrequency stimulation of the dorsal root ganglion (DRG) as a method for predicting targets for neuromodulation in patients with post amputation pain: A case series. Neuromodulation. 2017;20(7):708-18. doi: 10.1111/ner.12595. [PubMed: 28337820].

21. Deer TR, Levy RM, Kramer J, Poree L, Amirdelfan K, Grigsby E, et al. Comparison of paresthesia coverage of patient's pain: Dorsal root ganglion vs. Spinal cord stimulation. An accurate study sub-analysis. Neuromodulation. 2019;22(8):930-6. doi: 10.1111/ner.12920. [PubMed: 30624003].

22. Mekhail N, Deer TR, Kramer J, Poree L, Amirdelfan K, Grigsby E, et al. Paresthesia-free dorsal root ganglion stimulation: An accurate study sub-analysis. Neuromodulation. 2020;23(2):185-95. doi: 10.1111/ner.12942. [PubMed: 30861286].

23. Harrison C, Epton S, Bojanic S, Green AL, FitzGerald JJ. The efficacy and safety of dorsal root ganglion stimulation as a treatment for neuropathic pain: A literature review. Neuromodulation. 2018;21(3):225-33. doi:10.1111/ner.12685. [PubMed: 28960653].

24. Sivanesan E, Bicket MC, Cohen SP. Retrospective analysis of complications associated with dorsal root ganglion stimulation for pain relief in the FDA MAUDE database. Reg Anesth Pain Med. 2019;44(1):100-6. doi: 10.1136/rapm-2018-000007. [PubMed: 30640660]. [PubMed Central: PMC6363531].

25. Deer T, Pope J, Hunter C, Falowski S, Kapural L, Kramer J, et al. Safety analysis of dorsal root ganglion stimulation in the treatment of chronic pain. Neuromodulation. 2020;23(2):239-44. doi: 10.1111/ner.12941. [PubMed: 30861617]. [PubMed Central: PMC7065079].

26. Vuka I, Marcius T, Dosenovic S, Ferhatovic Hamzic L, Vucic K, Sapunar D, et al. Neuromodulation with electrical field stimulation of dorsal root ganglion in various pain syndromes: A systematic review with focus on participant selection. J Pain Res. 2019;12:80330. doi: 10.2147/JPR.S168814. [PubMed: 30881093]. [PubMed Central: PMC6398970].

27. Mol FMU, Roumen RMH. DRG spinal cord stimulation as solution for patients with severe pain due to anterior cutaneous nerve entrapment syndrome: A case series. Neuromodulation. 2018;21(3):317-9. doi: 10.1111/ner.12692. [PubMed: 28940994].

28. Hunter CW, Yang A. Dorsal root ganglion stimulation for chronic pelvic pain: A case series and technical report on a novel lead configuration. Neuromodulation. 2019;22(1):87-95. doi: 10.1111/ner.12801. [PubMed: 30067887].

29. Van Buyten JP, Smet I, Liem L, Russo M, Huygen F. Stimulation of dorsal root ganglia for the management of complex regional pain syndrome: A prospective case series. Pain Pract. 2015;15(3):208-16. doi: 10.1111/papr.12170. [PubMed: 24451048].

30. Piedade GS, Vesper J, Chatzikalfas A, Slotty PJ. Cervical and highthoracic dorsal root ganglion stimulation in chronic neuropathic pain. Neuromodulation. 2019;22(8):951-5. doi: 10.1111/ner.12916. [PubMed: 30620789].

31. Chapman KB, Groenen PS, Patel KV, Vissers KC, van Helmond N. T12 dorsal root ganglion stimulation to treat chronic low back pain: A case series. Neuromodulation. 2020;23(2):203-12. doi: 10.1111/ner.13047. [PubMed: 31588662].

32. Huygen FJPM, Liem L, Nijhuis H, Cusack W, Kramer J. Evaluating dorsal root ganglion stimulation in a prospective dutch cohort. Neuromod- ulation. 2019;22(1):80-6. doi: 10.1111/ner.12798. [PubMed: 30079622].

33. Morgalla MH, Fortunato M, Lepski G, Chander BS. Dorsal root ganglion stimulation (DRGS) for the treatment of chronic neuropathic pain: A single-center study with long-term prospective results in 62 cases. Pain Physician. 2018;21(1):E377-87. doi: 10.36076/ppj.2018.4.E377.

34. Levine AB, Steven DA, Parrent AG, MacDougall KW. Successful long-term nerve root stimulation for chronic neuropathic pain: A real world, single center Canadian experience. Pain Physician. 2017;20(2):95-106. [PubMed: 28158157].

35. Kallewaard JW, Nijhuis H, Huygen F, Wille F, Zuidema X, van de Minkelis J, et al. Prospective cohort analysis of DRG stimulation for failed back surgery syndrome pain following lumbar discectomy. Pain Pract. 2019;19(2):204-10. doi: 10.1111/papr.12734. [PubMed: 30269439].

36. Deer TR, Levy RM, Kramer J, Poree L, Amirdelfan K, Grigsby E, et al. Dorsal root ganglion stimulation yielded higher treatment success rate for complex regional pain syndrome and causalgia at 3 and 12 months: A randomized comparative trial. Pain. 2017;158(4):66981. doi: 10.1097/j.pain.0000000000000814. [PubMed: 28030470] [PubMed Central: PMC5359787].

37. Kallewaard JW, Edelbroek C, Terheggen M, Raza A, Geurts JW. A prospective study of dorsal root ganglion stimulation for nonoperated discogenic low back pain. Neuromodulation. 2020;23(2):196202. doi: 10.1111/ner.12937. [PubMed: 30821901].

38. Schu S, Gulve A, ElDabe S, Baranidharan G, Wolf K, Demmel W, et al. Spinal cord stimulation of the dorsal root ganglion for groin pain-a retrospective review. Pain Pract. 2015;15(4):293-9. doi: 10.1111/papr.12194. [PubMed: 24690212].

39. Hunter CW, Sayed D, Lubenow T, Davis T, Carlson J, Rowe J, et al. DRG FOCUS: A multicenter study evaluating dorsal root ganglion stimulation and predictors for trial success. Neuromodulation. 2019;22(1):6179. doi: 10.1111/ner.12796. [PubMed: 30085382].

40. Verrills P, Mitchell B, Vivian D, Cusack W, Kramer J. Dorsal root ganglion stimulation is paresthesia-independent: A retrospective study Neuromodulation. 2019;22(8):937-42. doi: 10.1111/ner.12921. [PubMed: 30701632].

41. Van Zundert J, Patijn J, Kessels A, Lame I, van Suijlekom H, van Kleef M. Pulsed radiofrequency adjacent to the cervical dorsal root ganglion in chronic cervical radicular pain: A double blind sham controlled randomized clinical trial. Pain. 2007;127(1-2):173-82. doi: 10.1016/j.pain.2006.09.002. [PubMed: 17055165].

42. Huygen F, Liem L, Cusack W, Kramer J. Stimulation of the L2-L3 dorsal root ganglia induces effective pain relief in the low back. Pain Pract. 2018;18(2):205-13. doi: 10.1111/papr.12591. [PubMed: 28486758].

43. Mol FMU, Roumen RM, Scheltinga MR. Comparing the efficacy of targeted spinal cord stimulation (SCS) of the dorsal root ganglion with conventional medical management (CMM) in patients with chronic post-surgical inguinal pain: The SMASHING trial. BMC Surg. 2018;18(1):18. doi: 10.1186/s12893-018-0349-8. [PubMed: 29587729]. [PubMed Central: PMC5872506].

44. Morgalla MH, de Barros Filho MF, Chander BS, Soekadar SR, Tatagiba M, Lepski G. Neurophysiological effects of dorsal root ganglion stimulation (DRGS) in pain processing at the cortical level. Neuromodulation. 2019;22(1):36-43. doi: 10.1111/ner.12900. [PubMed: 30561852].

45. Huygen FJPM, Kallewaard JW, Nijhuis H, Liem L, Vesper J, Fahey ME, et al. Effectiveness and safety of dorsal root ganglion stimulation for the treatment of chronic pain: A pooled analysis. Neuromodulation. 2020;23(2):213-21. doi: 10.1111/ner.13074. [PubMed: 31730273]. [PubMed Central: PMC7079258]. 